\title{
Collider Phenomenology of Techni-hadrons
}

\author{
Masafumi Kurachi* ${ }^{* \dagger}$ \\ Kobayashi-Maskawa Institute for the Origin of Particles and the Universe (KMI) \\ Nagoya University, Nagoya 464-8602, Japan \\ E-mail: kurachi@kmi.nagoya-u.ac.jp
}

\begin{abstract}
We consider the one-family technicolor model as the dynamical origin of the electroweak symmetry breaking. As low-energy degrees of freedom, we consider Techni-Pions (associated with the spontaneous breaking of global symmetry), Techni-Dilaton (which is identified as the $125 \mathrm{GeV}$ scalar discovered at the LHC) as well as Techni-Rho mesons as typical resonances expected to appear in such model. The effective Lagrangian is constructed based on the formulation of the Hidden Local Symmetry, and we discuss implications for collider phenomenology based on that Lagrangian.
\end{abstract}

KMI International Symposium 2013 on "Quest for the Origin of Particles and the Universe", 11-13 December, 2013

Nagoya University, Japan

\footnotetext{
* Speaker.

${ }^{\dagger}$ This talk is based on works in collaboration with S. Matsuzaki and K. Yamawaki.
} 


\section{Introduction}

The mystery of the origin of the masses of the fundamental particles is one of the most important issues to be revealed in elementary particle physics. Even though the property of the 125 $\mathrm{GeV}$ scalar boson discovered at the LHC seems to be quite consistent with that of the Higgs boson in the Standard Model (SM) so far, the existence of the hierarchy problem motivates us to look for an alternative scenario. Technicolor (TC) [1, 2, 3] is an attractive candidate for such alternatives, which has no hierarchy problem and provides the dynamical origin of the electroweak symmetry breaking. Also, phenomenologically viable TC models, so-called Walking TC (WTC) models [4, which incorporate approximately scale invariant dynamics with large mass anomalous dimension, $\gamma_{m} \simeq 1$, predict the existence of the light composite scalar boson as a pseudo Nambu-Goldstone (NG) boson associated with the spontaneous breaking of the approximate scale invariance. That is called the technidilaton (TD), which can be identified with the $125 \mathrm{GeV}$ boson discovered at the LHC [5]. Recent lattice studies [6] actually indicate that the existence of a light flavor-singlet bound state in theories of large $N_{f}$ QCD which have (near) conformal nature.

Here, as a concrete realization of the WTC, we consider the Farhi-Susskind one-family model [7], which consists of $N_{\mathrm{TC}}$ copies of a whole generation of the SM fermions, therefore the TC sector of the model is $S U\left(N_{\mathrm{TC}}\right)$ gauge theory with 8 fundamental Dirac fermions. The global chiral symmetry breaking pattern is then $S U(8)_{L} \times S U(8)_{R} / S U(8)_{V}$, resulting in the emergence of 63 NG bosons. Three of them are eaten by the SM weak gauge bosons, while 60 remain as physical states ("technipions"). Technipions obtain their masses through the explicit breaking effects (such as SM gauge interactions and extended TC four-fermion interactions), and become pseudo NG boson. Phenomenologies of these technipions are studied in detail in Ref. [8]. Here, we study the collider phenomenologies of another important techni-hadron, namely the techni-rho mesons, vector resonances expected to appear in models with strong dynamics.

\section{Effective lagrangian: scale invariant Hidden Local Symmetry}

To incorporate the techni-rho mesons, we apply the method of the Hidden Local Symmetry (HLS) [9, 10, 11, 12, 13] to the scale invariant chiral Lagrangian formulated in Ref. [5], which is based on the nonlinear realization of the manifold $G \times H_{\text {local }} / H=\left[S U(8)_{L} \times S U(8)_{R}\right] \times$ $S U(8)_{H} / S U(8)_{V}$ with the form made scale invariant by using a nonlinear field $\chi=e^{\phi / F_{\phi}}$ with the TD field $\phi$ :

$$
\begin{aligned}
\mathscr{L} & =\chi^{2}\left(F_{\pi}^{2} \operatorname{tr}\left[\hat{\alpha}_{\mu \perp}^{2}\right]+F_{\sigma}^{2} \operatorname{tr}\left[\hat{\alpha}_{\mu \| \mid}^{2}\right]\right)-\frac{1}{2 g^{2}} \operatorname{tr}\left[V_{\mu \nu}^{2}\right] \\
\hat{\alpha}_{\mu \perp, \|} & =\frac{D_{\mu} \xi_{R} \xi_{R}^{\dagger} \mp D_{\mu} \xi_{L} \xi_{L}^{\dagger}}{2 i} \\
D_{\mu} \xi_{L, R} & =\partial_{\mu} \xi_{L, R}-i V_{\mu} \xi_{L, R}+i \xi_{L, R} \mathscr{L}_{\mu}\left(\mathscr{R}_{\mu}\right) .
\end{aligned}
$$

Here, $\mathscr{L}_{\mu}$ and $\mathscr{R}_{\mu}$ are the SM gauge fields, while $V_{\mu}$ represents the HLS gauge field, which is identified as techni-rho mesons. The chiral-nonlinear fields $\left(\xi_{L}, \xi_{R}\right)$ are parametrized as

$$
\xi_{L, R}=e^{\frac{i \sigma}{F_{\sigma}}} e^{\mp \frac{i \pi}{F_{\pi}}}, \quad \pi=\pi^{A} X^{A}, \quad \sigma=\sigma^{A} X^{A},
$$


with the NG bosons associated with the HLS $(\sigma)$ and the chiral symmetry $(\pi)$. Here, $X^{A}$ is the generator of the $\mathrm{SU}(8)$ group, which can be expressed in such a way that it respects SM gauge representations:

$$
\begin{aligned}
& X_{\text {eaten }}^{i}=\frac{1}{2}\left(\begin{array}{l|l}
\tau^{i} \otimes 1_{3 \times 3} \mid & \tau^{i}
\end{array}\right), X_{P}^{i}=\frac{1}{2 \sqrt{3}}\left(\begin{array}{ll|l}
\tau^{i} \otimes 1_{3 \times 3} & \\
\hline & -3 \cdot \tau^{i}
\end{array}\right), X_{P}=\frac{1}{4 \sqrt{3}}\left(\begin{array}{l|l|}
1_{6 \times 6} \mid & -3 \cdot 1_{2 \times 2}
\end{array}\right), \\
& X_{\theta a}^{i}=\frac{1}{\sqrt{2}}\left(\begin{array}{c|c}
\tau^{i} \otimes \lambda_{a} \mid \\
\hline & 0
\end{array}\right), X_{\theta_{a}}=\frac{1}{2 \sqrt{2}}\left(\begin{array}{l|l|l}
1_{2 \times 2} \otimes \lambda_{a} \mid & 0
\end{array}\right) \text {, } \\
& X_{T c}^{(1) i}=\frac{1}{\sqrt{2}}\left(\frac{\mid \tau^{i} \otimes \xi_{c}}{\tau^{i} \otimes \xi_{c}^{\dagger}}\right), X_{T c}^{(2) i}=\frac{1}{\sqrt{2}}\left(\frac{\mid-i \tau^{i} \otimes \xi_{c}}{i \tau^{i} \otimes \xi_{c}^{\dagger}}\right) \text {, } \\
& X_{T c}^{(1)}=\frac{1}{2 \sqrt{2}}\left(\frac{\mid 1_{2 \times 2} \otimes \xi_{c}}{1_{2 \times 2} \otimes \xi_{c}^{\dagger}}\right), X_{T c}^{(2)}=\frac{1}{2 \sqrt{2}}\left(\frac{\mid-i \cdot 1_{2 \times 2} \otimes \xi_{c}}{i \cdot 1_{2 \times 2} \otimes \xi_{c}^{\dagger}}\right) .
\end{aligned}
$$

Here, $\tau^{i}=\sigma^{i} / 2, \xi_{c}$ being a three-dimensional unit vector in color space. Techni-rho mesons are expressed by the same $X^{A}$ as

$$
V_{\mu}=V_{\mu}^{A} X^{A}
$$

and SM gauge fields are expressed as follows:

$$
\begin{aligned}
& \mathscr{L}_{\mu}=2 g_{W} W_{\mu}^{i} X_{\text {eaten }}^{i}+\frac{2}{\sqrt{3}} g_{Y} B_{\mu} X_{P}+\sqrt{2} g_{s} G_{\mu}^{a} X_{\theta_{a}}, \\
& \mathscr{R}_{\mu}=2 g_{Y} B_{\mu}\left(X_{\text {eaten }}^{3}+\frac{1}{\sqrt{3}} X_{P}\right)+\sqrt{2} g_{s} G_{\mu}^{a} X_{\theta_{a}} .
\end{aligned}
$$

Interactions among techni-hadrons, techni-hadrons and gauge fields, as well as techni-hadrongauge-field mixing can be read off from the Lagrangian in Eq. (2.1) by expanding in $\pi$ and $\phi$.

\section{Techni-rho Production and Decay}

Now, we discuss production and decay of techni-rho mesons. Here, we take color-singlet isosinglet techni-rho $\left(\rho_{P}^{0}\right)$ as an example. ${ }^{1}$ As for the input parameters, we use $F_{\phi}=\left.F_{\phi}\right|_{\text {best }} \simeq v_{\mathrm{EW}} / 0.22$ (see Ref. [5]), $F_{\pi}=123 \mathrm{GeV}$ (to reproduce $v_{\mathrm{EW}}=246 \mathrm{GeV}$ ) and $g_{\rho}=g F_{\sigma}^{2}=\sqrt{2} M_{\rho} F_{\pi}$ (to achieve the vector meson dominance).

Techni-rho mesons are produced through the mixing with the SM gauge bosons which are produced by the Drell-Yan process. In Fig. 1 we show the production cross section of $\rho_{P}^{0}$ in $p p$ collision at the LHC as a function of $M_{\rho_{P}^{0}}$. The solid and dashed curves correspond to $\sqrt{s}=8$ and $14 \mathrm{TeV}$, respectively.

The decay properties of the techni-rho meson crucially depend on the mass spectrum of technihadrons. In Fig. 2 we plot the branching ratios of $\rho_{P}^{0}$ decay as functions of $M_{\rho_{P}^{0}}$ with assuming that the masses of the iso-singlet and the iso-triplet (color-triplet) techni-pions $\left(T_{0}, T_{i}\right)$ are $2 \mathrm{TeV}$. As one can see from the figure, above the threshold, i.e., $4 \mathrm{TeV}$ in this case, the $\rho_{P}^{0}$ dominantly decays to a techni-pion pair, while below the threshold, the decay property is rather rich. It is especially interesting to note that if the $\rho_{P}^{0}$ is heavier than $1 \mathrm{TeV}$, it mostly decays to the TD and $\gamma$. In the next section, we discuss this as a search channel of the techni-rho resonance in this model.

\footnotetext{
${ }^{1}$ More detailed studies, including other types of techni-rho mesons, can be found in Ref. [14].
} 


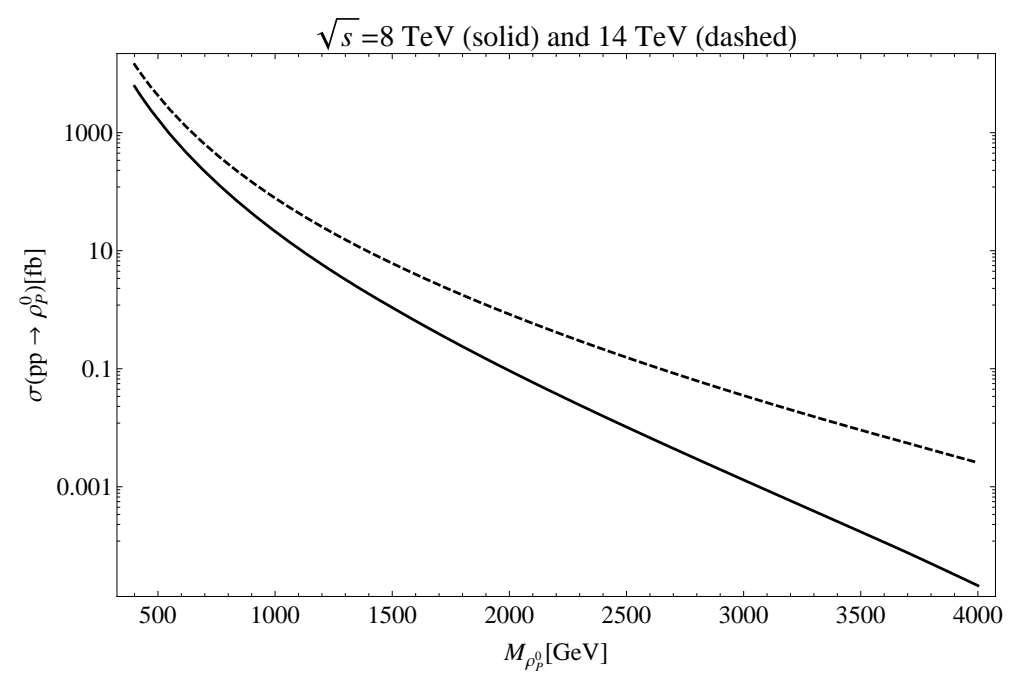

Figure 1: The LHC production cross section of $\rho_{P}^{0}$ in unit of fb as a function of $M_{\rho_{P}^{0}}$. The solid and dashed curves correspond to $\sqrt{s}=8$ and $14 \mathrm{TeV}$, respectively.

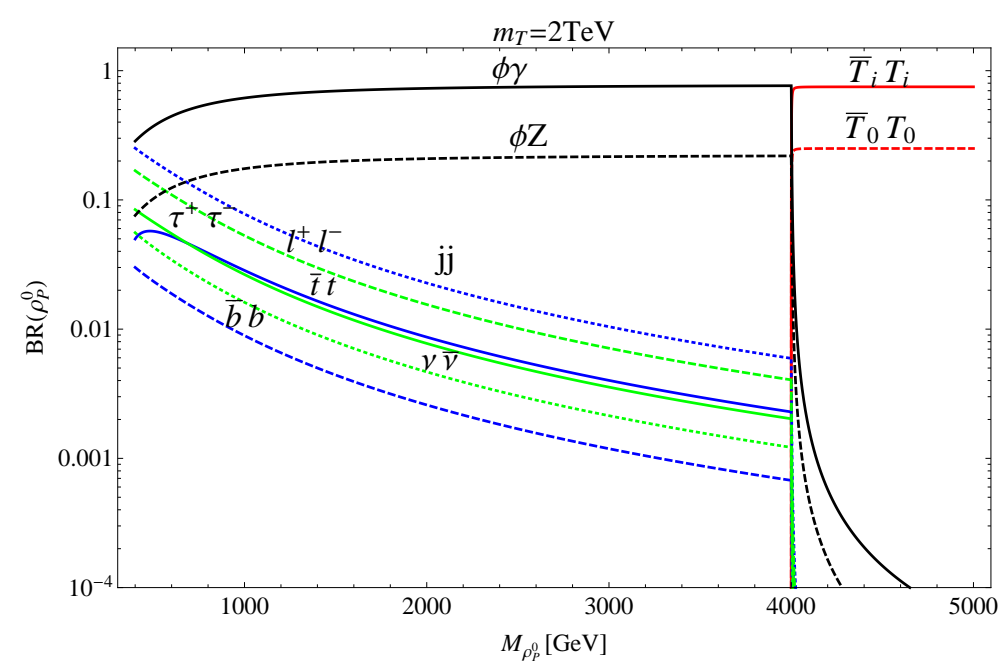

Figure 2: The branching fraction of $\rho_{P}^{0}$ with $M_{T_{0, i}}=2 \mathrm{TeV}$. " $j$ " in the figure represents the light-qurak $(u, d$, $s$ and $c$ ) jet.

\section{Constraints and Future Prospects}

In the previous section, we saw the production cross section of $\rho_{P}^{0}$ and the branching ratio of its decay. From these, together with the currently available LHC data, we can derive the constraints on the mass of $\rho_{P}^{0}$. A stringent constraint comes from the $Z^{\prime}$-type resonance search in di-lepton final states [15 16], and 95\% CL limit on the mass can be placed as $m_{\rho_{P}^{0}}>1.3 \mathrm{TeV}$.

As we mentioned in the previous section, possible search channel below the techni-pion pair production threshold would be $\rho_{P}^{0}$ decay into the TD $(\phi)$ and $\gamma$. The cross section of this channel in the mass range between the above lower bound and the techni-pion pair production threshold 


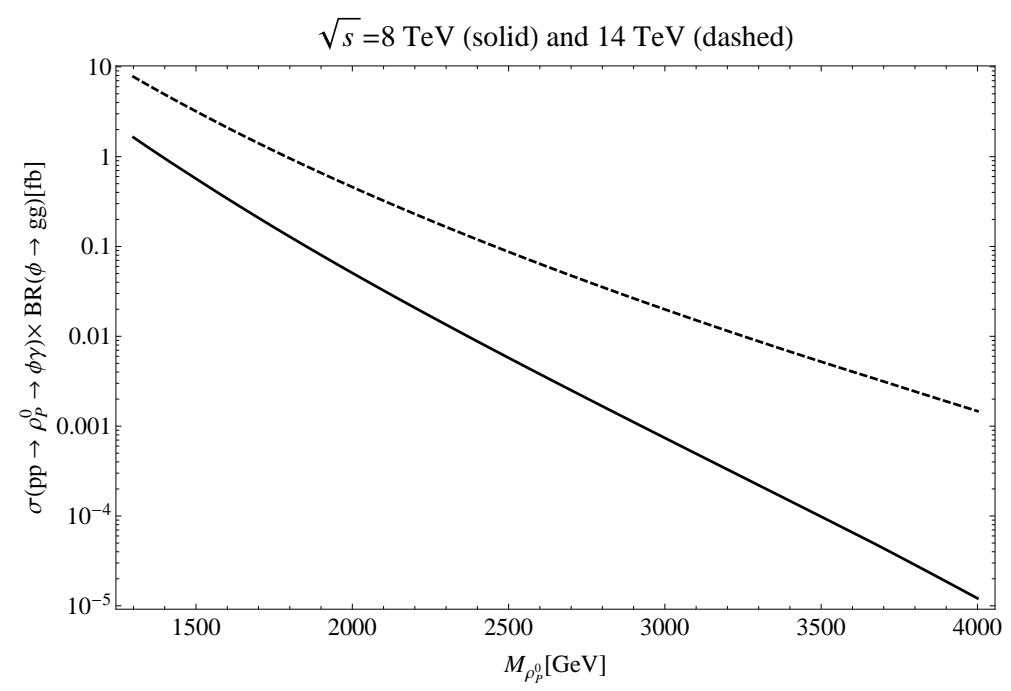

Figure 3: The total cross section $\sigma_{\mathrm{DY}}\left(p p \rightarrow \rho_{P}^{0} \rightarrow \phi \gamma\right) \times \mathrm{BR}(\phi \rightarrow g g)$ in unit of fb as a function of $M_{\rho_{P}^{0}}$. The solid and dashed curves correspond to $\sqrt{s}=8$ and $14 \mathrm{TeV}$, respectively. $\mathrm{BR}(\phi \rightarrow g g)=75 \%$ is assumed for the calculation.

is shown in Fig. 3. Here, we assumed that $\mathrm{BR}(\phi \rightarrow g g)=75 \%$. Detailed study on this process, including SM background estimation and detector simulations, will be presented elsewhere [17].

\section{Summary}

We considered the one-family technicolor model as the dynamical origin of the electroweak symmetry breaking. As low-energy degrees of freedom, we considered techni-pions (associated with the spontaneous breaking of global symmetry), techni-dilaton (which is identified as the 125 $\mathrm{GeV}$ scalar discovered at the LHC) as well as techni-rho mesons as typical resonances expected to appear in such model, and the effective Lagrangian is constructed based on the formulation of the Hidden Local Symmetry. The color-singlet iso-singlet techni-rho meson $\left(\rho_{P}^{0}\right)$ was taken as an example for the study of collider phenomenology of vector resonances. A constraint on the mass of $\rho_{P}^{0}$ from the currently available LHC data was estimated, and the TD- $\gamma$ final state was suggested as a search channel for future LHC experiment.

\section{References}

[1] S. Weinberg, Phys. Rev. D 13, 974 (1976);

[2] L. Susskind, Phys. Rev. D 20, 2619 (1979).

[3] For other reviews, see, e.g., K. Yamawaki, Lecture at 14th Symposium on Theoretical Physics, Cheju, Korea, July 1995, arXiv:hep-ph/9603293; C. T. Hill and E. H. Simmons, Phys. Rept. 381, 235 (2003) [Erratum-ibid. 390, 553 (2004)]; F. Sannino, Acta Phys. Polon. B40, 3533-3743 (2009).

[4] K. Yamawaki, M. Bando and K. Matumoto, Phys. Rev. Lett. 56, 1335 (1986); M. Bando, T. Morozumi, H. So and K. Yamawaki, Phys. Rev. Lett. 59, 389 (1987); M. Bando, K. Matumoto and K. Yamawaki, Phys. Lett. B 178, 308 (1986). 
[5] S. Matsuzaki and K. Yamawaki, Phys. Rev. D 85, 095020 (2012) [arXiv:1201.4722 [hep-ph]]; Phys. Rev. D 86, 035025 (2012) [arXiv:1206.6703 [hep-ph]]; Phys. Lett. B 719, 378 (2013) [arXiv:1207.5911 [hep-ph]]; S. Matsuzaki, arXiv:1304.4882 [hep-ph].

[6] Y. Aoki, T. Aoyama, M. Kurachi, T. Maskawa, K. Miura, K. -i. Nagai, H. Ohki, E. Rinaldi, A. Shibata, K. Yamawaki and T. Yamazaki (LatKMI Collaboration), arXiv:1309.0711 [hep-lat]; Y. Aoki, T. Aoyama, M. Kurachi, T. Maskawa, K. -i. Nagai, H. Ohki, E. Rinaldi, A. Shibata, K. Yamawaki and T. Yamazaki (LatKMI Collaboration), Phys. Rev. Lett. 111, 162001 (2013) [arXiv:1305.6006 [hep-lat]]; Z. Fodor, K. Holland, J. Kuti, D. Nogradi and C. H. Wong, arXiv:1401.2176 [hep-lat].

[7] E. Farhi and L. Susskind, Phys. Rept. 74, 277 (1981).

[8] J. Jia, S. Matsuzaki and K. Yamawaki, Phys. Rev. D 87, 016006 (2013) [arXiv:1207.0735 [hep-ph]].

[9] M. Bando, T. Kugo, S. Uehara, K. Yamawaki, and T. Yanagida, Phys. Rev. Lett. 54 (1985) 1215.

[10] M. Bando, T. Kugo, and K. Yamawaki, Nucl. Phys. B259 (1985) 493.

[11] M. Bando, T. Fujiwara, and K. Yamawaki, Prog. Theor. Phys. 79 (1988) 1140.

[12] M. Bando, T. Kugo, and K. Yamawaki, Phys. Rept. 164 (1988) 217-314.

[13] See also M. Harada and K. Yamawaki, Phys. Rept. 381 (2003) 1-233, hep-ph/0302103.

[14] M. Kurachi, S. Matsuzaki and K. Yamawaki, in preparation.

[15] The ATLAS Collaboration, ATLAS-CONF-2013-017.

[16] The CMS Collaboration, CMS-PAS-EXO-12-061.

[17] M. Kurachi, S. Matsuzaki, K. Terashi and K. Yamawaki, work in progress. 